\title{
28 Research Suare \\ The Karyotype Analysis of Sex Chromosome Mosaicism in Prenatal Diagnosis and Their Clinical Outcomes
}

\section{Shuang $\mathrm{Hu}$}

The center for genetics and prenatal diagnosis, the first affiliated hospital of Zhengzhou university, Jianshe Road Zhengzhou, Henan, China https://orcid.org/0000-0003-0310-9418

Ning Lu

The center for genetics and prenatal diagnosis, The First Affiliated Hospital of Zhengzhou University, No1 Jlanshe Road, Zhengzhou, Henan, China

\section{Xiangdong Kong ( $\square$ kongxd@263.net)}

The center for genetics and prenatal diagnosis, The First Affiliated Hospital of Zhengzhou University, Zhengzhou, Henan, China

\section{Research article}

Keywords: Karyotype analysis, Sex chromosome mosaicism, Amniocentisis, Prenatal diagnosis, Cytogenetic analysis

Posted Date: July 23rd, 2020

DOI: https://doi.org/10.21203/rs.3.rs-40267/v1

License: (9) This work is licensed under a Creative Commons Attribution 4.0 International License. Read Full License 


\section{Abstract}

Objective To analyze the karyotype of sex chromosome mosaicism in our prenatal diagnosis of 14034 pregnant women in their second trimester, and report the rate of sex chromosome mosaicism and their clinical outcomes.

Methods A retrospective analysis of cytogenetic studies of 14043 cases of pregnant women from the Genetic Counseling Clinic from May 2017 to January 2020 by amniocentesis, were performed.

Results A total of 46 cases of sex chromosome mosaicism were found, and the sex chromosome mosaicism rate was $0.328 \%$, mainly including four types of mosaicism: mos45,X/46, $X X(12)$; mos45,X/46,XY (11); mos47XXX(or XXY or XYY)/46XX(or XY)(11); and other types of complex abnormal karyotype mosaic(12). Among the 46 fetuses with sex chromosome mosaicism, the indications of prenatal diagnosis includes the numerical abnormality of sex chromosome by NIPT(23/46)凶the high risk of trisomy 21 by serum screening(12/46) \abnomal ultrosound(4/46), the advanced maternal age(age $\geq 35$ )(4/46), and the histories of abnormal pregnancy(3/46). According to the results of cytogenetic analysis and genetic counseling, the pregnant women would decide to continue or terminate their pregnancy.

Conclusion Prenatal cytogenetic diagnosis by amniocentesis is an accurate and convenient method and helps to avoid the delivery of fetuses with chromosomal diseases and reduce the risk of fetal malformation.

\section{Introduction}

It has been reported that sex chromosome mosaicism involving a numerical abnormality is the most common type of mosaicism ${ }^{1}$. The phenomenon of chromosomal mosaicism often occurs in prenatal diagnosis, including autosomal and sex chromosomal mosaicism. Sex chromosome abnormality may cause fetal sexual organ hypoplasia, usually accompanied by other organ structure aberrant and dysfunction ${ }^{2}$, or with mental retardation, mental and neurological disorders ${ }^{3}$. Therefore, it is very important to diagnosis fetus with chromosome aberrants.

Nowdays, it is a prevailing trend to perform prenatal screening and diagnosis, which could effectively reduce the born of children with abnormal chromosome. The karyotype analysis of amniotic fluid cells was the main strategy for detecting fetal chromosomal abnormalities, and was regard as the gold standard for cytogenetic diagnosis currently.

Here in our study, we retrospectively collect 14034 cases of pregnant women with specific prenatal diagnostic indications or of their own accord. Karyotype analysis of the amniotic fluid cells were performed. Of them, karyotype of 46 fetuses were sex chromosome mosaicism. Different proportions of mosaicism may have different effects on fetal prognosis, which makes increased difficulty in prenatal genetic counseling ${ }^{4,5}$. 
This study was approved by the Medical Ethics Committee in the First Affiliated Hospital of Zhengzhou University. All of the analysed samples were obtained with signed informed consent.

\section{Materials And Methods}

\section{Subjects}

Our study recruits 14034 pregnant women with the need of prenatal cytogenetic diagnosis who camed to the clicnic of the prenatal diagnosis center of the First Affiliated Hospital of Zhengzhou University between May 2017 and January 2020. Then amniotic fluid samples were successfully extracted and cultured, at last, all of the fetuses received karyotype diagnosis. All of the pregnant women performed amniocentesis with specific prenatal diagnostic indications or of their own accord. There are a variety of indications for genetic amnioticcentesis, including abnormal ultrasound finding, the numerical abnormality of sex chromosome by NIPT(Non-Invasive Prenatal Testing), the high risk of trisomy 21 by serum screening, abnormal ultrasound finding, the advanced maternal age(age $\geq 35$ ), and the histories of abnormal pregnancy. The pregnant women were at 17-24 weeks' gestation undergoing amniocentesis to get amniotic fluid samples.

\section{Methods}

Amniotic fluid specimens $(15 \mathrm{ml})$ was collected by amniocentesis under the guidance of ultrasonography, then centrifuged at $1500 \mathrm{r} / \mathrm{min}$ for 8 minutes. After discarding the supernatant, the remaining liquid was mixed and incubated with two $5 \mathrm{ml}$ of amniocyte culture medium (Gibco,USA, and Isreal). The cells grew in an incubator at $37^{\circ} \mathrm{C}$ and $5 \% \mathrm{CO} 2$ for about 9 days. When cell colonies reached more than 15 , these cells were collected followed by making sections and conventional Giemsa banding, then scanned by Zeiss Automated Nuclear Scanning System. For each sample, 5 mitotic figures were analyzed with 30 counts (abnormal karyotype with 100 counts). For these abnormal karyotypes, the second line cells were also managed as the first line.

\section{Results}

Out of the 14034 pregnant women recruited in our research, a total of 46 cases of sex chromosome mosaicism were found, and the sex chromosome mosaicism rate was $0.328 \%$, mainly including four types of mosaicism: $\operatorname{mos} 45, \mathrm{X} / 46, \mathrm{XX}(12)$; $\operatorname{mos} 45, \mathrm{X} / 46, \mathrm{XY}$ (11); $\operatorname{mos} 47 \mathrm{XXX}$ (or XXY or XYY)/46XX(or XY) (11); and other types of abnormal karyotype mosaic(12). The detailed information is in the Table 1. 
Table 1

Karyotype analysis of 46 cases with sex chromosome mosaicism

\begin{tabular}{|c|c|c|c|}
\hline No. & karyotype & indications & $\begin{array}{l}\text { Follow- } \\
\text { ups }\end{array}$ \\
\hline 1 & $45 \otimes X[37] / 46 \otimes X X[63]$ & $\begin{array}{l}\text { high risk of trisomy } 21 \text { by serum } \\
\text { screening }\end{array}$ & abort \\
\hline 2 & $45 \otimes X[26] / 46 \rrbracket X X[74]$ & $\begin{array}{l}\text { the numerical abnormality of sex } \\
\text { chromosome by NIPT }\end{array}$ & abort \\
\hline 3 & $45 \rrbracket X[47] / 46 \rrbracket X X[53]$ & $\begin{array}{l}\text { the numerical abnormality of sex } \\
\text { chromosome by NIPT }\end{array}$ & abort \\
\hline 4 & $45 \rrbracket X[63] / 46 \rrbracket X X[37]$ & $\begin{array}{l}\text { high risk of trisomy } 21 \text { by serum } \\
\text { screening }\end{array}$ & abort \\
\hline 5 & $45 \otimes X[54] / 46 \otimes X X[46]$ & $\begin{array}{l}\text { the numerical abnormality of sex } \\
\text { chromosome by NIPT }\end{array}$ & abort \\
\hline 6 & $45 \rrbracket X[56] / 46 \rrbracket X X[44]$ & age $\geq 35$ & abort \\
\hline 7 & $45 \bigotimes X[83] / 46 \rrbracket X X[17]$ & $\begin{array}{l}\text { the numerical abnormality of sex } \\
\text { chromosome by NIPT }\end{array}$ & abort \\
\hline 8 & $45 \rrbracket X[82] / 46 \rrbracket X X[18]$ & $\begin{array}{l}\text { the numerical abnormality of sex } \\
\text { chromosome by NIPT }\end{array}$ & abort \\
\hline 9 & $45 \otimes X[49] / 46 \rrbracket X X[51]$ & age $\geq 35$ & abort \\
\hline 10 & $45 \rrbracket X[5] / 46 \rrbracket X X[95]$ & $\begin{array}{l}\text { the numerical abnormality of sex } \\
\text { chromosome by NIPT }\end{array}$ & continue \\
\hline 11 & $45 \bigotimes X[16] / 46 \bigotimes X X[84]$ & $\begin{array}{l}\text { high risk of trisomy } 21 \text { by serum } \\
\text { screening }\end{array}$ & $\begin{array}{l}\text { lose } \\
\text { contact }\end{array}$ \\
\hline 12 & $45 \rrbracket X[61] / 46 \rrbracket X X[39]$ & the histories of abnormal pregnancy & abort \\
\hline 13 & $45 \rrbracket X[24] / 46 \rrbracket X Y[76]$ & the histories of abnormal pregnancy & continue \\
\hline 14 & $45 \rrbracket X[4] / 46 \rrbracket X Y[96]$ & $\begin{array}{l}\text { high risk of trisomy } 21 \text { by serum } \\
\text { screening }\end{array}$ & continue \\
\hline 15 & $45 \rrbracket X[46] / 46 \rrbracket X Y[54]$ & abnormal ultrosound & continue \\
\hline 16 & $45 \rrbracket X[77] / 46 \llbracket X Y[23]$ & $\begin{array}{l}\text { high risk of trisomy } 21 \text { by serum } \\
\text { screening }\end{array}$ & abort \\
\hline 17 & $45 \rrbracket X[54] / 46 \rrbracket X Y[46]$ & $\begin{array}{l}\text { the numerical abnormality of sex } \\
\text { chromosome by NIPT }\end{array}$ & abort \\
\hline 18 & $45 \bigotimes X[66] / 46 \bigotimes X Y[34]$ & $\begin{array}{l}\text { the numerical abnormality of sex } \\
\text { chromosome by NIPT }\end{array}$ & $\begin{array}{l}\text { lose } \\
\text { contact }\end{array}$ \\
\hline 19 & $45 \otimes X[17] / 46 \bigotimes X Y[83]$ & $\begin{array}{l}\text { the numerical abnormality of sex } \\
\text { chromosome by NIPT }\end{array}$ & abort \\
\hline 20 & $45 \rrbracket X[12] / 46 \rrbracket X Y[88]$ & high risk of trisomy 21 by serum & continue \\
\hline
\end{tabular}


$21 \quad 45 \rrbracket X[12] / 46 \bigotimes X Y[88]$

$22 \quad 45 \llbracket X[14] / 46 \bigotimes X Y[86]$

$2345 \rrbracket X[27] / 46 \rrbracket X Y[73]$

$24 \quad 47 \otimes X X X[7] / 46 \otimes X X[93]$

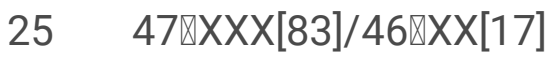

$2647 \rrbracket X X X[23] / 46 \otimes X X[77]$

$27 \quad 47 \rrbracket X X X[6] 46 \rrbracket X Y[94]$

$2847 \rrbracket X X Y[38] / 46 \rrbracket X Y[62]$

$29 \quad 47 \rrbracket X X Y[6] / 46 \rrbracket X Y[94]$

$30 \quad 47 \rrbracket X X Y[38] / 46 \rrbracket X Y[62]$

$31 \quad 47 \rrbracket X X Y[13] / 46 \rrbracket X Y[87]$

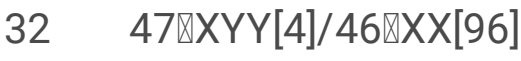

$3347 \rrbracket X Y Y[87] / 46 \rrbracket X X[13]$

$34 \quad 47 \bigotimes X Y Y[18] / 46 \rrbracket X Y[82]$

$35 \quad 45 \rrbracket X[53] / 47 \rrbracket X X X[47]$

$3645 \otimes X[70] / 47 \rrbracket X Y Y[30]$

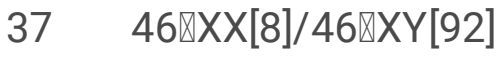

$3846 \rrbracket X Y[95] / 46 \rrbracket X X[5]$

$3945 \otimes X[80] / 46 \rrbracket X \bigotimes i(X)(q 10)[20]$

40

$45 \rrbracket X[61] / 46 \rrbracket X \bigotimes i(X)(q 10)[39]$

41 the numerical abnormality of sex

chromosome by NIPT

the numerical abnormality of sex

chromosome by NIPT

high risk of trisomy 21 by serum screening

age $\geq 35$

age $\geq 35$

the numerical abnormality of sex

chromosome by NIPT

the numerical abnormality of sex

chromosome by NIPT

high risk of trisomy 21 by serum screening

the numerical abnormality of sex chromosome by NIPT

the histories of abnormal pregnancy

abnormal ultrosound

abnormal ultrosound

the numerical abnormality of sex chromosome by NIPT

high risk of trisomy 21 by serum screening

the numerical abnormality of sex chromosome by NIPT

the numerical abnormality of sex chromosome by NIPT

high risk of trisomy 21 by serum screening

abnormal ultrosound

the numerical abnormality of sex

chromosome by NIPT

the numerical abnormality of sex chromosome by NIPT

the numerical abnormality of sex chromosome by NIPT lose contact

continue

continue

continue

continue

continue

abort

continue

continue

abort

continue

abort

continue

continue

abort

continue

abort

abort

abort

abort

abort $\operatorname{inv}(9)(\mathrm{p} 11 \mathrm{q} 13)[67]$ 


\begin{tabular}{|c|c|c|c|}
\hline 42 & $\begin{array}{l}\text { 45区X囚inv(9)(p11q13)[53]/46冈XY区 } \\
\text { inv(9)(p11q13)[47] }\end{array}$ & $\begin{array}{l}\text { the numerical abnormality of sex } \\
\text { chromosome by NIPT }\end{array}$ & abort \\
\hline 43 & 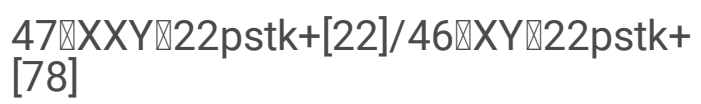 & $\begin{array}{l}\text { the numerical abnormality of sex } \\
\text { chromosome by NIPT }\end{array}$ & abort \\
\hline 44 & $\begin{array}{l}45 \rrbracket X[18] / 46 \rrbracket X X \rrbracket t(3 ; 7)(q 21 ; q 22) \\
{[5] / 46 \rrbracket X X[77]}\end{array}$ & $\begin{array}{l}\text { the numerical abnormality of sex } \\
\text { chromosome by NIPT }\end{array}$ & abort \\
\hline 45 & $47 \rrbracket X X X[42] / 48 \llbracket X X X X[2] / 46 \llbracket X X[56]$ & $\begin{array}{l}\text { high risk of trisomy } 21 \text { by serum } \\
\text { screening }\end{array}$ & abort \\
\hline 46 & $\begin{array}{l}\text { 45खX[43]/46冈X『dic(X;X)(q28;q28) } \\
{[6] / 46 \rrbracket X X[51]}\end{array}$ & $\begin{array}{l}\text { high risk of trisomy } 21 \text { by serum } \\
\text { screening }\end{array}$ & abort \\
\hline
\end{tabular}

Among the 46 fetuses with sex chromosome mosaicism, the indications of prenatal diagnosis are different, 23 cases were the numerical abnormality of sex chromosome by NIPT(23/46), 12 pregnant women had the high risk of trisomy 21 by serum screening(12/46), the other indications like abnomal ultrosound(4/46), the advanced maternal age(age $\geq 35)(4 / 46)$, and the histories of abnormal pregnancy(3/46), are less than the former two indications. The detailed proportion is shown as Fig. 1 indicated obviously.

\section{Follow ups}

For all the 46 cases with mosaic sex chromosome karyotype, 27 pregnant women had the abortion, 16 pregnant women had a normal delivery, 3 cases lost contact. The overall rate of pregnancy termination is $58.7 \%$.

\section{Discussion}

Chromosome abnormality are common genetics disorders causing spontaneous abortion, fetus aberration or birth defects ${ }^{6,7}$. However, there are no effective methods to treat this kind of abnormal chromosome disorders. Nowdays with the rapid development of technology, amniocentesis and subsequent karyotype analysis is the gold standard for prenatal disgnosis, which is an important way to confirm and prevent aberrant pregnancies ${ }^{8,9}$. Chromosome mosaicism would cause varying degrees of fetal abnormalities according to the mosaic propotion. Sex chromosome mosaicism will lead to hermaphroditism and psychiatric disorders ${ }^{10}$.

Early work indicated that sex chromosome mosaic is the most common type of mosaicism, which involved either a loss or gain of one sex chromosome, with the proportion of nearly a half of all kinds of mosaicism, including sex chromosome mosaicism, autosomal mosaicism and marker chromosome mosaicism ${ }^{1}$. Out of the 14046 cases of fetal karyotype analysis, 46 fetus were found sex chromosome 
mosaicism, the sex chromosome mosaicism proportion is $0.327 \%$, which is a little lower than that of others' research in China reported in $2018^{11}$.

For all these 46 cases, their prenatal diagnostic indications are different, a half of them were the numerical abnormality of sex chromosome by NIPT, which could increase the sex chromosome mosaicism proportion among general pregnant women, because of the high accuracy rate of NIPT. $26 \%$ of the 46 pregnant women were grouped into high-risk serum screening, other high risk indications are less than or only $8.7 \%$. In lights with these findings, we would summarize that of the proportion of sex chromosome mosaicism is less than $\mathbf{0 . 3 2 7 \%}$ of all the pregnant women in China. So when they had high risk in the numerical abnormality of sex chromosome by NIPT, they must perform the amniocentesis to get accurate cytogenetic diagnosis to exclude sex chromosome abnormality due to its definite pathogenicity.

Among these mosaic cases, $45, X$ (Turner Syndrome) is the most common chromosome abnormality. 31 out of 46 were $45, X$ mosaicism, which usually causes infertility, cardiac and kidney malformation, and ear and hearing problems ${ }^{12}$. Sex chromosome trisomies mosaicism, including 47,XXY, 47,XXX, 47,XYY, that is an extra $\mathrm{X}$ or $\mathrm{Y}$ chromosome in karyotype, are less than $45, \mathrm{X}$ mosaicism.

$47, \mathrm{XXY}$ (Klinefelter syndrome) usually leads to varying clinical manifestation, like infertility, small testes, and problems with psychological development, lower IQ, and delayed speech development ${ }^{13}$. $47, \mathrm{XXX}$ (Triple X) girls are at high risks for difficulties in language, neuromotor and learning skills and behavior problems ${ }^{14} .47, \mathrm{XYY}$ usually shows worldwide attention deficit hyperactivity disorder symptoms, comprised language development, phychotic disorder ${ }^{15,16}$. Among all these mosaicisms, only one fetus have 48,XXXX mosaicism karyotype. It was reported that girls with tetrasomy X had dysmorphic facial features, varying degrees of cognitive dysfunction, speech and language disorders and skeletal and connective tissue disorders ${ }^{17}$. There are sex chromosomal or autosomal structure abnormality for 6 cases, including inversion, translocation, isochromosome, dicentric $\mathrm{X}$ chromosome. One case is the short arm of chromosome 22 increased with the length of the body stalk (22sptk+), which is normal chromosome variation.

It has been indicated that Turner syndrome, Klinefelter syndrome, and $45, \mathrm{X}$ mosaicism were main factors associated with parental dicisions to terminate an affected pregnancy ${ }^{18}$. In our study, most pregnant women prenatal diagnosed 45,X/46,XX mosaicisms choose to abort their pregnancies, except one case with $45, X[5] / 46, X X[95]$ karyotype, because of high proportion of normal karyotype. However, as regard to $45, \mathrm{X} / 46, \mathrm{XY}$ karyotype,when the proportion of $46, \mathrm{XY}$ was higher than $80 \%$, almost all women choose to continue the pregnancy. This may be due to the traditional Chinese demand for boys. Especially for one pedigree the abnormal karyotype was $45, \mathrm{X} / 46, \mathrm{XX}$, and the mosaic proportion was $46 \%$ and $54 \%$, respectively, the family urged to continue the pregnancy even the doctor adviced them to abort.

If the karyotypes of the three sex chromosomes $(47, \mathrm{XXX}, 47, \mathrm{XXY}, 47, \mathrm{XYY})$ are chimeric with the normal two sex chromosomes $(46, X X, 46, X Y)$, pregnant women usually choose to induce labor,but when the proportion 
of normal karyotypes are higher than $80 \%$,pregnant women would continue the pregnancies. When the karyotypes of the chimeras are all abnormal karyotypes, that is, either the number or structure of sex chromosomes are abnormal or the structure of autosomes is abnormal, pregnant women would choose to induce labor. When there are three types of karyotypes in the chimera, pregnant women choose to induce labor. Three pedigrees lost contact, and we could not know their choices.

In lights with these findings, we would suggest the fetus with mosaic sex chromosome karyotype abort their pregnancies if the abnormal karyotype was higher than $80 \%$.

However, there are many potential factors associated with parental decisions regarding abnormal sex chromosome pregnancy in our research, we just did a simple follow-up, more studies need to be explored.

Concluding, if the pregnant women have clear incidences of prenatal diagnosis, they had better to follow the doctors' advices to conduct prenatal cytogenetic diagnosis. Prenatal cytogenetic diagnosis by amniocentesis is an accurate and convenient method and helps to avoid the delivery of fetuses with chromosomal diseases and reduce the risk of fetal malformation.

\section{Declarations}

\section{Ethics approval and consent to participate:}

This study was approved by the Medical Ethics Committee in the First Affiliated Hospital of Zhengzhou University. All of the analysed samples were obtained with signed informed consent.

\section{Consent for publication:}

All of the analysed samples were obtained with signed informed consent.

\section{Availability of data and material:}

All data generated or analysed during this study are included in this published article and its supplementary information files.

\section{Competing interests:}

The authors declare that they have no competing interests

\section{Funding:}


This work was supported by grants from the Hospital Internal Funding from the First Affiliated Hospital of Zhengzhou University.

\section{Authors' contributions:}

XK and SH conceived the study, SH and NL summarized all the data and involved in histological analysis, XK provided expertise for data interpretation and suggestions for manuscript preparation. SH wrote the manuscript.

\section{Acknowledgements:}

Not applicable

\section{References}

1. Hsu LY, Yu MT, Richkind KE, et al: Incidence and significance of chromosome mosaicism involving an autosomal structural abnormality diagnosed prenatally through amniocentesis: a collaborative study. Prenat Diagn. 1996; 16(1):1-28.

2. Tartaglia NR, Ayari N, Hutaff-Lee $\mathrm{C}$, et al: Attention-deficit hyperactivity disorder symptoms in children and adolescents with sex chromosome aneuploidy: $X X Y, X X X, X Y Y$, and $X X Y Y$. Journal of developmental and behavioral pediatrics : JDBP. 2012; 33(4):309-18.

3. Lee NR, Wallace GL, Adeyemi El, et al: Dosage effects of $X$ and $Y$ chromosomes on language and social functioning in children with supernumerary sex chromosome aneuploidies: implications for idiopathic language impairment and autism spectrum disorders. Journal of child psychology and psychiatry, and allied disciplines. 2012; 53(10):1072-81.

4. Lucas-Herald AK, Cann F, Crawford L, et al: The outcome of prenatal identification of sex chromosome abnormalities. Archives of disease in childhood Fetal and neonatal edition. 2016; 101(5):F423-7.

5. Stanescu D, van Lemputten R, Frans A, et al: Evaluation of lung function indices for bronchodilator trials. Results of a cross-over study of fenoterol. Respiration; international review of thoracic diseases. 1976; 33(1):1-8.

6. Shen JD, Sun FX, Qu DY, et al: [Chromosome abnormality rate and related factors of spontaneous abortion in early pregnancy]. Zhonghua fu chan ke za zhi. 2019; 54(12):797-802.

7. Del Carmen Nogales $M$, Bronet F, Basile $N$, et al: Type of chromosome abnormality affects embryo morphology dynamics. Fertility and sterility. 2017; 107(1):229-35 e2.

8. Wang J, Chen L, Zhou C , et al: Prospective chromosome analysis of 3429 amniocentesis samples in China using copy number variation sequencing. American journal of obstetrics and gynecology. 2018; 219(3):287 e1- e18. 
9. Pin-Jung C, Pai-Chi T, Zhu Y, et al: Noninvasive Prenatal Diagnostics: Recent Developments Using Circulating Fetal Nucleated Cells. Current obstetrics and gynecology reports. 2019; 8(1):1-8.

10. Green T, Flash S, Reiss AL: Sex differences in psychiatric disorders: what we can learn from sex chromosome aneuploidies. Neuropsychopharmacology : official publication of the American College of Neuropsychopharmacology. 2019; 44(1):9-21.

11. Li Xian-zheng XL, Hu Jing-jing, Liu Yuan, Li Xing: The Rate of Sex Chromosomal Mosaicism in Prenatal Diagnosis and Clinical outcomes J Inc Reported Health/Fam Plan. 2018; 4(37):292-6.

12. Kubba H, Smyth A, Wong SC, et al: Ear health and hearing surveillance in girls and women with Turner's syndrome: recommendations from the Turner's Syndrome Support Society. Clinical otolaryngology : official journal of ENT-UK ; official journal of Netherlands Society for Oto-RhinoLaryngology \& Cervico-Facial Surgery. 2017; 42(3):503-7.

13. Linden MG, Bender BG: Fifty-one prenatally diagnosed children and adolescents with sex chromosome abnormalities. American journal of medical genetics. 2002; 110(1):11-8.

14. Afshan A: Triple X syndrome. JPMA The Journal of the Pakistan Medical Association. 2012; 62(4):392-4.

15. Ross JL, Roeltgen DP, Kushner $H$, et al: Behavioral and social phenotypes in boys with $47, X Y Y$ syndrome or 47,XXY Klinefelter syndrome. Pediatrics. 2012; 129(4):769-78.

16. van Rijn S: A review of neurocognitive functioning and risk for psychopathology in sex chromosome trisomy (47,XXY, 47,XXX, 47, XYY). Current opinion in psychiatry. 2019; 32(2):79-84.

17. Jayaraman $D$, Carvalho KS, Hasbani DM: A case report of hypersomnia in tetrasomy $X$ improved with medical therapy. Clin Case Rep. 2018; 6(5):893-5.

18. Jeon KC, Chen LS, Goodson P: Decision to abort after a prenatal diagnosis of sex chromosome abnormality: a systematic review of the literature. Genetics in medicine : official journal of the American College of Medical Genetics. 2012; 14(1):27-38.

\section{Figures}




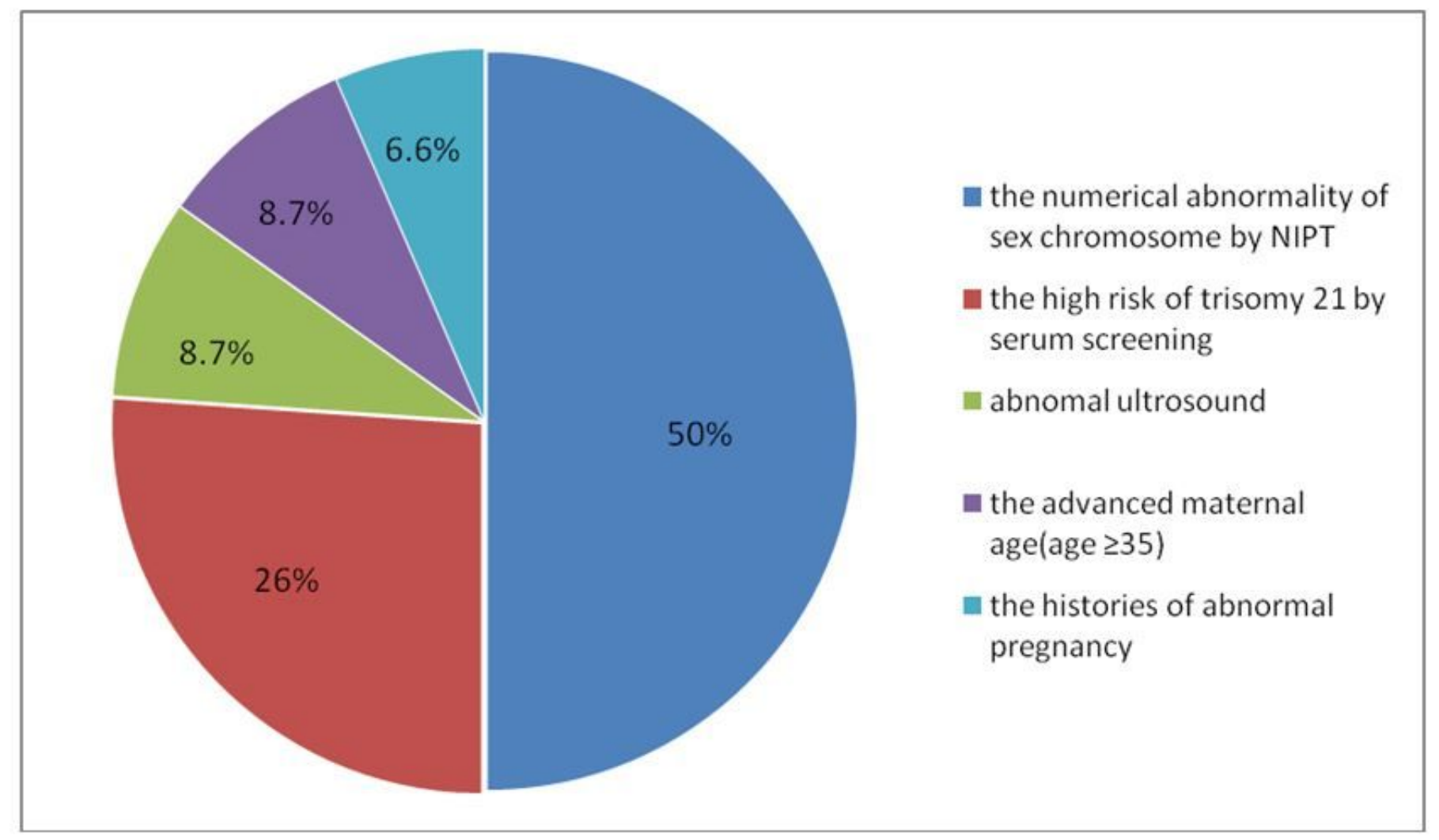

Figure 1

the proportion of different indications of prenatal diagnosis 